Kragujevac Journal of Mathematics

Volume 38(2) (2014), Pages 315-320.

\title{
ON THE DOMINATION AND TOTAL DOMINATION NUMBERS OF CAYLEY SUM GRAPHS OVER $\mathbb{Z}_{n}$
}

\author{
M. AMOOSHAHI ${ }^{1}$ AND B. TAERI ${ }^{2}$
}

\begin{abstract}
Let $G$ be a finite Abelian group and $S$ be a subset of $G$. The Cayley sum graph $\mathrm{Cay}^{+}(G, S)$ of $G$ with respect to $S$ is a graph whose vertex set is $G$ and two vertices $g$ and $h$ are joined by an edge if and only if $g+h \in S$. In this paper, we prove some basic facts on the domination and total domination numbers of Cayley sum graphs. Then, we find the sharp bounds for domination number of Cay ${ }^{+}\left(\mathbb{Z}_{n}, S\right)$, where $S=\{1,2, \ldots, k\}$ and $n, k$ are positive integers with $1 \leq k \leq(n-1) / 2$.
\end{abstract}

\section{INTRODUCTION}

Let $G$ be a finite Abelian group and $S$ be a subset of $G$. The Cayley sum graph $\mathrm{Cay}^{+}(G, S)$ is the graph having the vertex set $G$ and the edge set $\{\{g, h\} \mid g, h \in$ $G, g+h \in S\}$. If $S$ is a multiset, then $\mathrm{Cay}^{+}(G, S)$ contains multiple edges, and if there exists $g \in G$ with $2 g \in S$, then the edge $\{g, g\}$ is a semiedge, i.e. an edge with one endpoint. Unlike a loop, a semiedge contributes just one to both the valency of its endpoint and the corresponding diagonal entry of the adjacency matrix. With this convention, $\mathrm{Cay}^{+}(G, S)$ is a regular graph with valency $|S|$.

Cayley sum graphs also are known under names addition Cayley graphs $[5,6,7]$, addition graphs [2] and sum graphs [3]. The study of Cayley sum graphs has been the object of some papers, for example, independence number [1], hamiltonicity $[2,6]$, expander properties [3], clique number [4] and connectivity [5].

Recall that a set $D \subseteq V$ of vertices in a graph $\mathcal{G}=(V, E)$ is called a dominating set if every vertex $v \in V$ is either an element of $D$ or is adjacent to an element of $D$. A dominating set $D$ is a minimal dominating set if no proper subset is a dominating set. The domination number $\gamma(\mathcal{G})$ of a graph $\mathcal{G}$ is the minimum cardinality of a dominating set in $\mathcal{G}$ and the corresponding dominating set is called a $\gamma$-set.

Key words and phrases. Cayley sum graph, (Total) dominating set, (Total) domination number. 2010 Mathematics Subject Classification. 05C25.

Received: May 28, 2014

Accepted: November 12, 2014. 
A set $T \subseteq V(\mathcal{G})$ is a total dominating set if every vertex in $V(\mathcal{G})$ is adjacent to a vertex in $T$. The total domination number of a graph $\mathcal{G}$ denoted by $\gamma_{t}(\mathcal{G})$ is the minimum cardinality of all total dominating sets. Clearly, $\gamma(\mathcal{G}) \leq \gamma_{t}(\mathcal{G})$.

In this paper, we give some results on the (total) domination number of Cayley sum graphs. Then, we find some (total) dominating sets and (total) domination numbers in graph $\mathrm{Cay}^{+}\left(\mathbb{Z}_{n},\{1,2, \ldots, k\}\right)$, where $n$ and $k$ are positive integers with $1 \leq k \leq(n-1) / 2$.

\section{Main Results}

Hereafter, $n$ is a positive integer, $\mathbb{Z}_{n}=\{0,1,2, \ldots, n-1\}$ and + stands for modulo $n$ addition in $\mathbb{Z}_{n}$. If $g$ and $h$ are adjacent in $\operatorname{Cay}^{+}(G, S)$, i.e. $g+h \in S$, we write $g \sim h$. Also, $N(g)=\{h \in G \mid g+h \in S\}$ and $N[g]=N(g) \cup\{g\}$ denote the set of open neighbours and closed neighbours of $g$, respectively.

In what follows, we prove some basic facts on the (total) domination number of Cayley sum graphs.

Theorem 2.1. Let $G$ be a finite Abelian group of order $n$ and $S$ be a subset of $G$. Then,

$$
\left\lceil\frac{n}{|S|+1}\right\rceil \leq \gamma\left(\mathrm{Cay}^{+}(G, S)\right) \leq n-|S|+1
$$

Proof. First, we consider the lower bound. If $g \in G$ is a vertex such that $2 g \in S$, then $g$ can dominate $|S|$ vertices. If $g \in G$ is a vertex such that $2 g \notin S$, then $g$ can dominate itself and $|S|$ other vertices. So, $\gamma\left(\mathrm{Cay}^{+}(G, S)\right) \geq\left\lceil\frac{n}{|S|+1}\right\rceil$.

For the upper bound, if there exists $g \in G$ such that $2 g \notin S$, then $g$ dominates $N[g]$ vertices and the vertices in $G \backslash N[g]$ dominate themselves. Hence, $G \backslash N(g)$ is a dominating set of cardinality $n-|S|$. If $2 G \subseteq S$, then $g$ dominates $N[g]=N(g)$ vertices, for every $g \in G$, and the vertices in $G \backslash N(g)$ dominate themselves. Thus, $G \backslash N(g) \cup\{g\}$ is a dominating set of cardinality $n-|S|+1$. Therefore, $\gamma\left(\mathrm{Cay}^{+}(G, S)\right) \leq$ $n-|S|+1$.

The bounds for $\gamma\left(\mathrm{Cay}^{+}(G, S)\right)$ obtained in Theorem 2.1, can not be reduced in general. More specifically, the lower bound is attained for the graph $\mathcal{G}_{1}=\mathrm{Cay}^{+}\left(\mathbb{Z}_{4},\{1,3\}\right)$ and the upper bound is attained for the graph $\mathcal{G}_{2}=\mathrm{Cay}^{+}\left(\mathbb{Z}_{5},\{0,1,2,4\}\right)$.

Theorem 2.2. Let $G$ be a finite Abelian group of order $n$ and $S$ be a subset of $G$, such that $\mathrm{Cay}^{+}(G, S)$ has no component of size 1 . Then,

$$
\gamma_{t}\left(\mathrm{Cay}^{+}(G, S)\right) \leq n-|S|+1 \text {. }
$$

Proof. Let $g \in G$ and put $X=G \backslash N[g]$. If $X=\emptyset$, then $|S|=n-1$ or $|S|=n$. Thus, $\gamma_{t}\left(\mathrm{Cay}^{+}(G, S)\right)=2$ or $\gamma_{t}\left(\mathrm{Cay}^{+}(G, S)\right)=1$. If $X \neq \emptyset$, then by considering $I$ as the set of all components of size $1{\text { of } \mathrm{Cay}^{+}(G, S)[X] \text {, the subgraph of Cay }}^{+}(G, S)$ induced by $X$, we have two cases; 
Case 1: $I=\emptyset$. If $2 g \in S$, then $X \cup\{g\}$ is a total dominating set and so, $\gamma_{t}\left(\mathrm{Cay}^{+}(G, S)\right) \leq|X|+1=n-|S|+1$. If $2 g \notin S$, then $X \cup\{g\} \cup\{h\}$ is a total dominating set, where $h \in N(g)$, and thus $\gamma_{t}\left(\mathrm{Cay}^{+}(G, S)\right) \leq|X|+2=n-|S|+1$.

Case 2: $I \neq \emptyset$. We denote by $C(I)$ a subset of $N(g)$ with the smallest cardinality such that each $i \in I$ is adjacent to an element of $C(I)$. Clearly, $|C(I)| \leq|I|$. Then, $(X \backslash I) \cup C(I) \cup\{g\}$ is a total dominating set and so, $\gamma_{t}\left(\mathrm{Cay}^{+}(G, S)\right) \leq|X \backslash I|+$ $|C(I)|+1 \leq|X|+1 \leq n-|S|+1$.

In [6], Lev proved that if $S$ is a subset of a finite Abelian group $G$, then $\operatorname{Cay}^{+}(G, S)$ is connected if and only if $S$ is not contained in a coset of a proper subgroup of $G$, except, perhaps, for the non-zero coset of a subgroup of index 2. In the following theorem, we find a bound for the total domination number of a connected Cayley sum graph.

Theorem 2.3. Let $G$ be a finite Abelian group of order $n$ and $S$ be a subset of $G$ of size less than $n-1$ such that $\mathrm{Cay}^{+}(G, S)$ is connected. If $S \cap 2 G=\emptyset$, then $\gamma_{t}\left(\mathrm{Cay}^{+}(G, S)\right) \leq n-|S|$ and if $S \cap 2 G \neq \emptyset$, then $\gamma_{t}\left(\mathrm{Cay}^{+}(G, S)\right) \leq n-|S|+1$.

Proof. Let $g, X$ and $I$ be as in the proof of Theorem 2.2. Since $|S| \leq n-2, X \neq \emptyset$. We have two cases. If $I=\emptyset$, by connectivity of $\mathrm{Cay}^{+}(G, S)$, some $x \in X$ is adjacent to some $h \in N(g)$. Let $V$ and $\Delta$ be the vertex set and maximum degree of the component of $\mathrm{Cay}^{+}(G, S)[X]$ which contains $x$, respectively. By Theorem $2.2, \mathrm{Cay}^{+}(G, S)[V]$ has a total dominating set $T$ of cardinality at most $|V|-\Delta+1$. If $\Delta=1$ and $2 x \in S$, then $|V|=1$ and so $\{g, h\} \cup(X \backslash V)$ is a total dominating set. Thus, $\gamma_{t}\left(\mathrm{Cay}^{+}(G, S)\right) \leq$ $2+|X|-|V|$. Now, if $2 g \in S$, then $|X|=n-|S|$ and so $\gamma_{t}\left(\mathrm{Cay}^{+}(G, S)\right) \leq n-|S|+1$, else $|X|=n-|S|-1$ and so $\gamma_{t}\left(\mathrm{Cay}^{+}(G, S)\right) \leq n-|S|$. If $\Delta=1$ and $2 x \notin S$, then $|V|=2$ and so $\{g, h, x\} \cup(X \backslash V)$ is a total dominating set. Thus, similar to above discussion, $\gamma_{t}\left(\mathrm{Cay}^{+}(G, S)\right) \leq n-|S|+1$ or $\gamma_{t}\left(\mathrm{Cay}^{+}(G, S)\right) \leq n-|S|$. If $\Delta>1$, then $\{g, h\} \cup T \cup(X \backslash V)$ is a total dominating set and thus $\gamma_{t}\left(\mathrm{Cay}^{+}(G, S)\right) \leq$ $2+|T|+|X|-|V| \leq|X|-\Delta+3=|X|+1+(2-\Delta) \leq|X|+1$. Again, by similar discussion mentioned above, $\gamma_{t}\left(\mathrm{Cay}^{+}(G, S)\right) \leq n-|S|+1$ or $\gamma_{t}\left(\mathrm{Cay}^{+}(G, S)\right) \leq n-|S|$. The proof of the remaining case $(I \neq \emptyset)$ is similar to that of Theorem 2.2.

Now, we find some results on $\gamma\left(\mathrm{Cay}^{+}\left(\mathbb{Z}_{n}, S\right)\right)$ and $\gamma_{t}\left(\mathrm{Cay}^{+}\left(\mathbb{Z}_{n}, S\right)\right)$, where $S=$ $\{1,2, \ldots, k\}$ and $1 \leq k \leq(n-1) / 2$.

Theorem 2.4. Let $\mathcal{G}=\mathrm{Cay}^{+}\left(\mathbb{Z}_{n},\{1,2, \ldots, k\}\right)$, where $n, k$ are positive integers with $1 \leq k \leq(n-1) / 2$. Then, $\left\lceil\frac{n}{k+1}\right\rceil \leq \gamma(\mathcal{G}) \leq\left\lceil\frac{n}{k}\right\rceil$.

Proof. Consider the set $D=\{0, k, 2 k, \ldots,(l-1) k\}$, where $l=\lceil n / k\rceil$. We claim that $D$ is a dominating set of $\mathcal{G}$. For an arbitrary vertex $c \in \mathbb{Z}_{n}$, if $c \in S$, then $c \sim 0$. If $c \in \mathbb{Z}_{n} \backslash S$, then by division algorithm, we have $n-c=i k+r$ such that $0 \leq r \leq k-1$ and $0 \leq i \leq l-2$. So, $c+(i+1) k=c+i k+k=n-r+k \equiv k-r<k(\bmod n)$ and thus $c \sim(i+1) k \in D$ which implies that $c \in N((i+1) k)$ and $D$ is a dominating set. Therefore, by Theorem 2.1, $\left\lceil\frac{n}{k+1}\right\rceil \leq \gamma(\mathcal{G}) \leq\left\lceil\frac{n}{k}\right\rceil$. 
The bounds are given in Theorem 2.4 are sharp. Consider two Cayley sum graphs $\mathcal{G}_{1}=\mathrm{Cay}^{+}\left(\mathbb{Z}_{15},\{1,2,3,4\}\right)$ and $\mathcal{G}_{2}=\mathrm{Cay}^{+}\left(\mathbb{Z}_{8},\{1,2,3\}\right)$. The lower and upper bound is attained for $\mathcal{G}_{1}$ and $\mathcal{G}_{2}$, respectively.

Let $\varphi: \mathcal{G} \longrightarrow \mathcal{H}$ be a graph isomorphism and $D$ be a $\gamma$-set of $\mathcal{G}$. One can easily show that $\varphi(D)$ is a dominating set of $\mathcal{H}$. So, $\gamma(\mathcal{H}) \leq|\varphi(D)|=\gamma(\mathcal{G})$. Similarly, by considering $\varphi^{-1}: \mathcal{H} \longrightarrow \mathcal{G}$, we have $\gamma(\mathcal{G}) \leq \gamma(\mathcal{H})$. Therefore we have the following lemma.

Lemma 2.1. If two graphs are isomorphic, then their domination numbers are equal.

Theorem 2.5. Let $n$ and $r$ be positive integers. Then,

$$
\gamma\left(\mathrm{Cay}^{+}\left(\mathbb{Z}_{n}, A_{i}\right)\right)=\gamma\left(\mathrm{Cay}^{+}\left(\mathbb{Z}_{n}, A_{i+2 r}\right)\right)
$$

where $A_{i}=\{i, i+1, \ldots, i+k-1\}$, for every $i, k \in \mathbb{Z}_{n}$.

Proof. Define $\varphi: \mathrm{Cay}^{+}\left(\mathbb{Z}_{n}, A_{i}\right) \longrightarrow \mathrm{Cay}^{+}\left(\mathbb{Z}_{n}, A_{i+2 r}\right)$ given by $\varphi(m)=m+r$. Clearly, $\varphi$ is a bijection. It remains to show that $\varphi$ is adjacent preserving. In $\mathrm{Cay}^{+}\left(\mathbb{Z}_{n}, A_{i}\right)$, if $a \sim b$, then $\varphi(a)+\varphi(b)=a+b+2 r \in A_{i}+2 r=A_{i+2 r}$ and so in $\mathrm{Cay}^{+}\left(\mathbb{Z}_{n}, A_{i+2 r}\right)$ we have $\varphi(a) \sim \varphi(b)$. Therefore, $\varphi$ is a graph isomorphism. Now, Lemma 2.1 implies the result.

The converse of above theorem does not necessary hold. For example, let $n=6$, $A_{1}=\{1,2,3\}$ and $A_{2}=\{2,3,4\}$. We have $\gamma\left(\mathrm{Cay}^{+}\left(\mathbb{Z}_{6}, A_{1}\right)\right)=\gamma\left(\mathrm{Cay}^{+}\left(\mathbb{Z}_{6}, A_{2}\right)\right)$, but there is no $r \in \mathbb{Z}_{6}$ such that $A_{2}=A_{1+2 r}$.

Theorem 2.6. Let $n$ and $r$ be two positive integers such that $(r, n)=1$. If $A$ is a subset of $\mathbb{Z}_{n}$, then

$$
\gamma\left(\mathrm{Cay}^{+}\left(\mathbb{Z}_{n}, A\right)\right)=\gamma\left(\mathrm{Cay}^{+}\left(\mathbb{Z}_{n}, r A\right)\right) .
$$

Proof. Define $\varphi: \mathrm{Cay}^{+}\left(\mathbb{Z}_{n}, A\right) \longrightarrow \mathrm{Cay}^{+}\left(\mathbb{Z}_{n}, r A\right)$ given by $\varphi(m)=r m$. Clearly, $\varphi$ is a bijection. It suffices to show that $\varphi$ is adjacent preserving. In $\mathrm{Cay}^{+}\left(\mathbb{Z}_{n}, A\right)$, if $a \sim b$, then $\varphi(a)+\varphi(b)=r a+r b=r(a+b) \in r A$ and so in $\mathrm{Cay}^{+}\left(\mathbb{Z}_{n}, r A\right)$ we have $\varphi(a) \sim \varphi(b)$. Therefore, $\varphi$ is a graph isomorphism and thus, by Lemma 2.1, the result holds.

Theorem 2.7. Let $S=\{1,2, \ldots, k\}$ be a subset of $\mathbb{Z}_{n}$, where $n \in \mathbb{N}$ and $k \in \mathbb{Z}_{n}$. Then,

$$
\gamma_{t}\left(\mathrm{Cay}^{+}\left(\mathbb{Z}_{n}, S\right)\right)=\left\lceil\frac{n}{k}\right\rceil
$$

Proof. Let $T_{0}$ be a $\gamma_{t}$-set of $\mathrm{Cay}^{+}\left(\mathbb{Z}_{n}, S\right)$ such that $\gamma_{t}\left(\mathrm{Cay}^{+}\left(\mathbb{Z}_{n}, S\right)\right)=\left|T_{0}\right|$. Every element in $T_{0}$ is adjacent to at most $k-1$ vertices in $V(\mathcal{G}) \backslash T_{0}$. Also, by definition, every vertex in $T_{0}$ is adjacent to some vertices in $T_{0}$. Thus, $\left|T_{0}\right|(k-1)+\left|T_{0}\right| \geq n$ and so, $\left|T_{0}\right| k \geq n$ which implies that $\left|T_{0}\right|=\gamma_{t}(\mathcal{G}) \geq\left\lceil\frac{n}{k}\right\rceil$.

Now, we claim that $T=\left\{0, k, 2 k, \ldots,\left(\left\lceil\frac{n}{k}\right\rceil-1\right) k\right\}$ is a total dominating set. By Theorem 2.4, it is enough to show that every element of $T$ is adjacent to some elements of $T$. Put $l:=\left\lceil\frac{n}{k}\right\rceil$. So, $n=(l-1) k+r$, where $0 \leq r<k$. For $i=1,2, \ldots, l-1$, 
let $i k$ be an arbitrary element of $T$. Since $l k=n+k-r \equiv k-r \leq k(\bmod n)$ and $(i k)+((l-i) k)=l k$, we conclude that $i k \sim(l-i) k$. Also, $0 \sim k$. So, $T$ is a total dominating set. Thus $\gamma_{t}(\mathcal{G}) \leq|T|=\left\lceil\frac{n}{k}\right\rceil$, as required.

Theorem 2.8. Let $S=\{1,2, \ldots, k\} \subseteq \mathbb{Z}_{n}$ and $T=\left\{0, k, 2 k, \ldots,\left(\left\lceil\frac{n}{k}\right\rceil-1\right) k\right\}$, where $k \in \mathbb{Z}_{n}$ and $n \in \mathbb{N}$. Then, $T_{i}=T+i$ is a total dominating set of $\mathrm{Cay}^{+}\left(\mathbb{Z}_{n}, S\right)$, for all $i \in\{0,1, \ldots, k-1\}$.

Proof. Let $c$ be an arbitrary vertex in $\operatorname{Cay}^{+}\left(\mathbb{Z}_{n}, S\right)$. If $c \in\{1,2, \ldots, n-i-1\}$, then $n-c-i=t k+r$, where $0 \leq r \leq k-1$ and so $c+(t+1) k+i=n+k-r \equiv k-r \in S$ $(\bmod n)$ implies that $c$ is adjacent to $(t+1) k+i \in T_{i}$. If $c=n-i$, then $c+i+k=$ $n-i+i+k=n+k \equiv k \in S(\bmod n)$ implies that $c$ is adjacent to $i+k \in T_{i}$. If $c \in\{n-i+1, \ldots, n\}$, then $c$ is adjacent to $i \in T_{i}$, because $c+i \in S$. Therefore, $T_{i}$ is a total dominating set, for every $i \in\{0,1, \ldots, k-1\}$.

Consider the notations used in Theorem 2.8. Let $c$ be an arbitrary vertex in $\mathrm{Cay}^{+}\left(\mathbb{Z}_{n}, S\right)$. Then, there exist integers $t$ and $i$ such that $c=t k+i$ and $0 \leq i \leq k-1$. Clearly, $c \in T_{i}$, where $0 \leq i \leq k-1$. This shows that every vertex in $\mathrm{Cay}^{+}\left(\mathbb{Z}_{n}, S\right)$ is contained in some $\gamma_{t}$-sets.

Theorem 2.9. Let $\mathrm{Cay}^{+}\left(\mathbb{Z}_{n}, S_{1}\right)$ and $\mathrm{Cay}^{+}\left(\mathbb{Z}_{m}, S_{2}\right)$ be two connected graphs, where $S_{1} \subseteq \mathbb{Z}_{n}$ and $S_{2} \subseteq \mathbb{Z}_{m}$. Then,

$$
\gamma_{t}\left(\mathrm{Cay}^{+}\left(\mathbb{Z}_{n}, S_{1}\right)\right) \gamma_{t}\left(\mathrm{Cay}^{+}\left(\mathbb{Z}_{m}, S_{2}\right)\right) \geq \gamma_{t}\left(\mathrm{Cay}^{+}\left(\mathbb{Z}_{n} \times \mathbb{Z}_{m}, S_{1} \times S_{2}\right)\right) .
$$

Proof. Suppose that $T_{1}$ and $T_{2}$ are $\gamma_{t}$-sets of Cay ${ }^{+}\left(\mathbb{Z}_{n}, S_{1}\right)$ and $\mathrm{Cay}^{+}\left(\mathbb{Z}_{m}, S_{2}\right)$, respectively. We claim that $T=T_{1} \times T_{2}$ is a $\gamma_{t}$-set of $\mathrm{Cay}^{+}\left(\mathbb{Z}_{n} \times \mathbb{Z}_{m}, S_{1} \times S_{2}\right)$. For an arbitrary element $(x, y) \in \mathbb{Z}_{n} \times \mathbb{Z}_{m}$, there exist $t_{1} \in T_{1}$ and $t_{2} \in T_{2}$ such that $x$ is adjacent to $t_{1}$ and $y$ is adjacent to $t_{2}$. Therefore, $(x, y)$ is adjacent to $\left(t_{1}, t_{2}\right)$ in $T$ and thus, $T$ is a total dominating set of $\mathrm{Cay}^{+}\left(\mathbb{Z}_{n} \times \mathbb{Z}_{m}, S_{1} \times S_{2}\right)$.

Note that, in Theorem 2.9, the equality can be hold. For example, consider $\mathrm{Cay}^{+}\left(\mathbb{Z}_{2},\{1\}\right)$ and $\mathrm{Cay}^{+}\left(\mathbb{Z}_{3},\{1,2\}\right)$. By Theorem 2.7, we have $\gamma_{t}\left(\mathrm{Cay}^{+}\left(\mathbb{Z}_{2},\{1\}\right)\right)=$ $\gamma_{t}\left(\mathrm{Cay}^{+}\left(\mathbb{Z}_{3},\{1,2\}\right)\right)=2$. On the other hand $\gamma_{t}\left(\mathrm{Cay}^{+}\left(\mathbb{Z}_{2} \times \mathbb{Z}_{3},\{(1,1),(1,2)\}\right)\right)=4$, as desired.

Acknowledgment: The authors gratefully thank the referee for the valuable remarks and suggestions for the improvement of the paper.

\section{REFERENCES}

[1] N. Alon, Large sets in finite fields are sumsets, J. Number Theory 126(1) (2007), 110-118.

[2] B. Cheyne, V. Gupta and C. Wheeler, Hamilton cycles in addition graphs, Rose-Hulman Undergraduate Math. Journal 1 (4) (2003).

[3] F. R. K. Chung, Diameters and eigenvalues, J. Amer. Math. Soc. 2(2) (1989), 187-196.

[4] B. Green, Counting sets with small sumset, and the clique number of random Cayley graphs, Combinatorica 25 (2005), 307-326.

[5] D. Grynkiewicz, V. F. Lev and O. Serra, Connectivity of addition Cayley graphs, J. Comin. Theory Ser. B 99 (2009), 202-217. 
[6] V. F. Lev, Sums and differences along Hamiltonian cycles, Discrete Math. 310 (2010), 575-584.

[7] D. Sinha, P. Garg and A. Singh, Some properties of unitary addition Cayley graphs, NNTDM $\mathbf{1 7}(3)(2011), 49-59$.

${ }^{1}$ Department of Mathematical Sciences, ISFAHAN UNIVERSITY OF TECHNOLOGY, ISFAHAN 84156-83111, IRAN

E-mail address: m.amooshahi@math.iut.ac.ir

${ }^{2}$ Department of Mathematical Sciences, ISFAHAN UNIVERSITY OF TECHNOLOGY, ISFAHAN 84156-83111, IRAN

E-mail address: b.taeri@cc.iut.ac.ir 